\title{
Avulsion fracture of the extensor carpi radialis longus in a rugby player: a case report
}

\author{
D Jena, K A Giannikas, R Din
}

\begin{abstract}
Avulsion fracture of the base of the second metacarpal is an unusual injury, and the cause in the few cases reported in the literature was a fall on a volarly flexed wrist. A case of this rare injury suffered in a sport related accident by a semiprofessional rugby player is reported. It was treated with open reduction and internal fixation after failure of conservative management.

(Br J Sports Med 2001;35:133-135)
\end{abstract}

Keywords: avulsion fracture; metacarpal; wrist; rugby

\section{Case report}

A twenty six year old semiprofessional rugby player attended the accident and emergency department complaining of pain at the dorsum of his right dominant hand. Two days previously, he had sustained a forceful hyperflexion injury of his wrist in a heavy head on tackle. Anteroposterior and lateral radiographs of his wrist were obtained but were interpreted as normal (fig 1).

On review at the fracture clinic the following day, a minimally tender swelling was noticed on the dorsum of his hand at the base of the second metacarpal. The swelling was noticed to be of bony consistency and could be reduced on full dorsiflexion of the wrist. On closer inspection of the $x$ ray films, a fracture line was suspected at the level of the base of the second metacarpal. Oblique views failed to confirm the fracture. On clinical grounds, it was decided to treat the patient as having sustained an avulsion fracture of the insertion of the extensor carpi radialis in the base of the second metacarpal.

The patient was treated by applying a below elbow volar slab with the wrist in full dorsiflexion. At review after one week, the swelling was noticed to have recurred and the patient was taken to theatre. Under general anaesthetic, the hand was rotated under image intensification to establish the exact anatomy of the avulsed fragment (fig 2). Closed reduction was attempted but failed to restore the fragment. The fracture was subsequently explored through a longitudinal $5 \mathrm{~cm}$ incision over the osseous prominence, and the fragment was anatomically reduced and fixed with two smooth $\mathrm{K}$ wires (fig 3). The wrist was immobilised with a below elbow cast in neutral position for four weeks, after which the wires were removed (fig 4) and active physiotherapy was initiated.

Twelve weeks after the injury the patient had regained full range of movement of the wrist and complete function of the hand without any
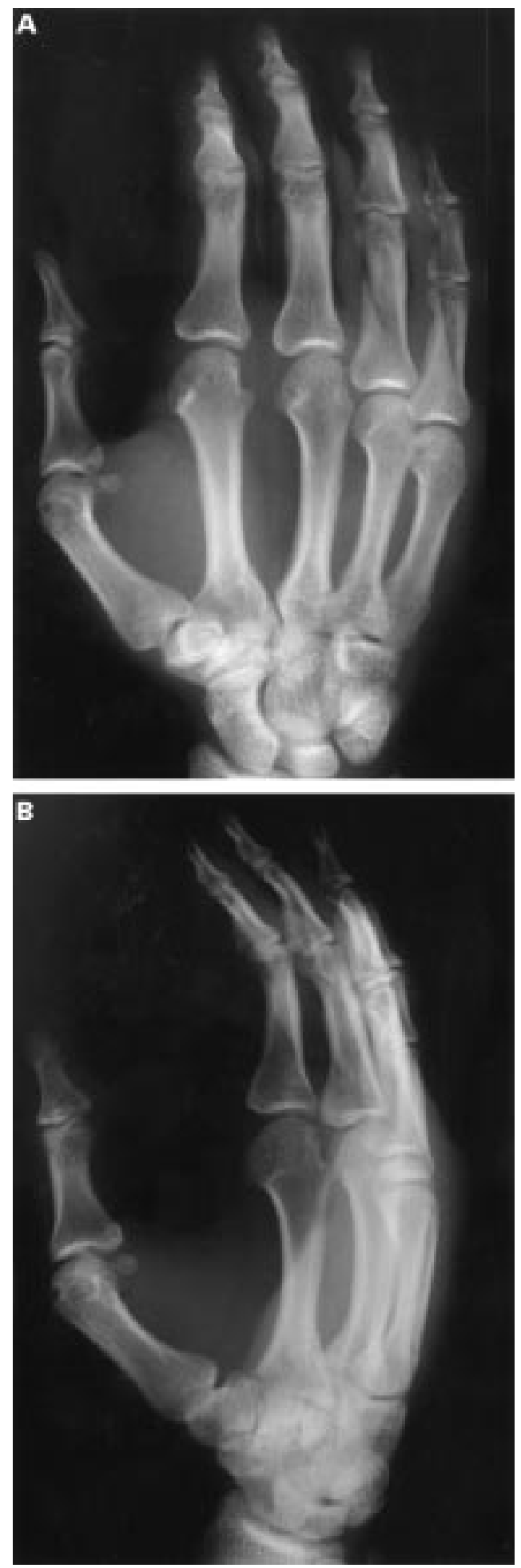

Figure 1 Anteroposterior (A) and lateral (B) radiographs of the wrist of the rugby player who had sustained a hyperflexion injury. 


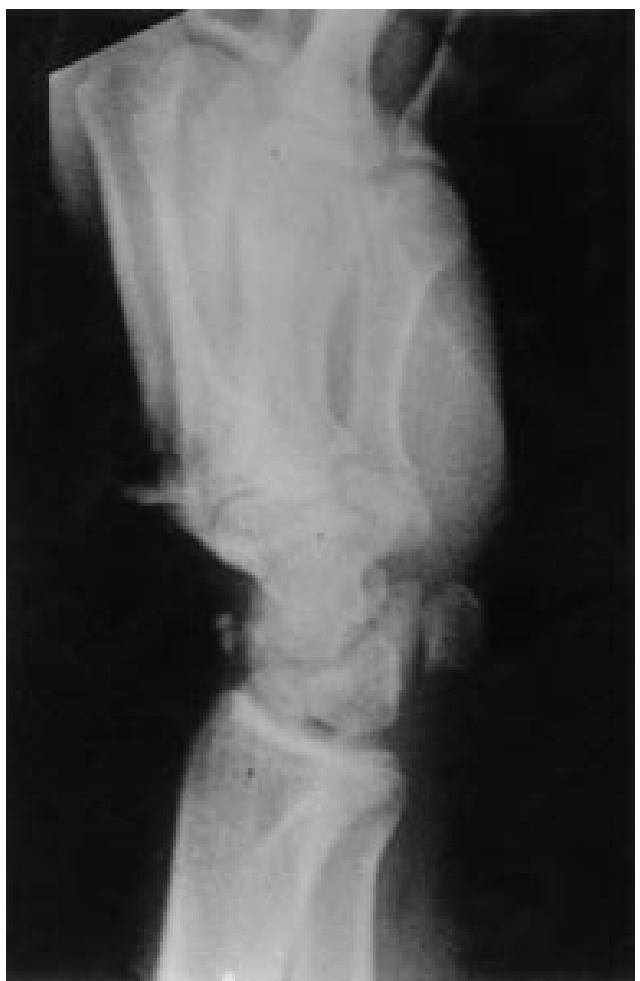

Figure 2 Use of image intensification to establish the exact anatomy of the avulsed fragment.

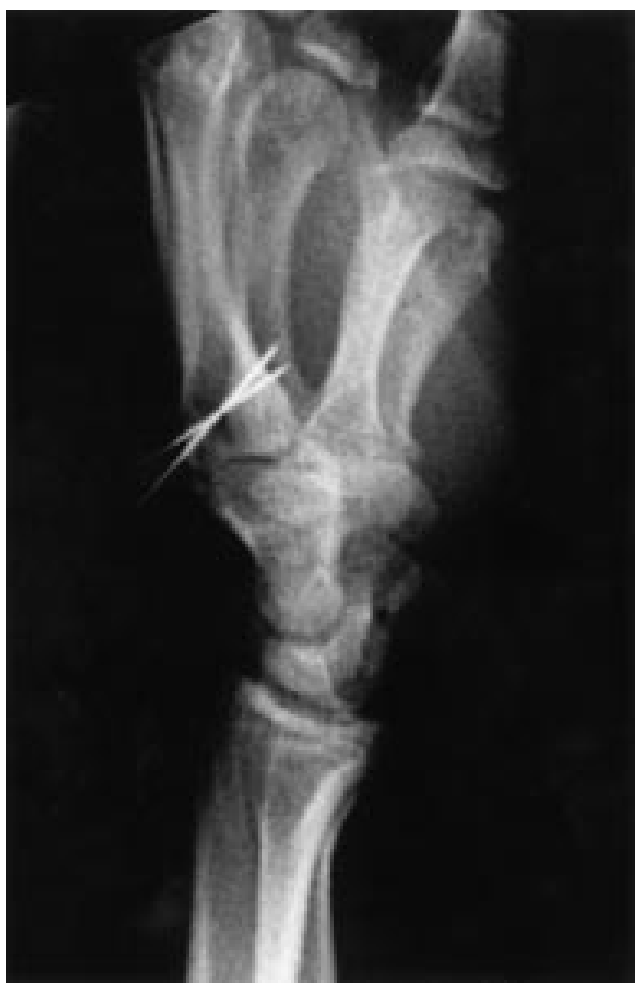

Figure 3 Radiograph of wrist after anatomical reduction and fixation of the avulsed fragment with two smooth $K$ wires.

pain. He was advised to refrain from contact sports for a further six weeks.

\section{Discussion}

Only seven cases have been described in the literature of avulsion fractures of the extensor carpi radialis longus at its insertion over the

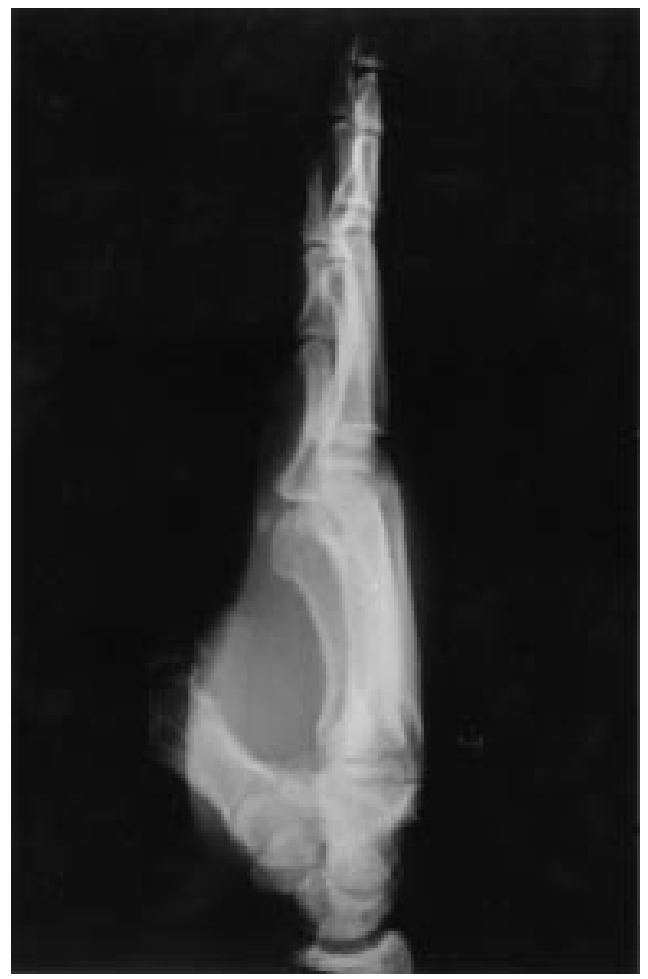

Figure 4 Radiograph of the wrist four weeks after surgery when the $K$ wires had been removed.

base of the second metacarpal. ${ }^{1-6}$ This injury can be understood if one considers the anatomy of the second carpometacarpal joint.

\section{ANATOMY}

The base of the second metacarpal forms a fork-like structure, with the opening towards the carpus, and articulates with the trapezium, the trapezoid, the capitate, and the third metacarpal. The tendon of the extensor carpi radialis longus is inserted at the dorsum of the base of the second metacarpal forming a "hoodlike" structure over the carpometacarpal articulation. Furthermore the base of the second metacarpal provides attachment to a capsular ligamental complex on both volar and dorsal aspects of the carpus. This arrangement means that only limited flexion and extension are possible around the second carpometacarpal joint. $^{7}$

MECHANISM AND MANAGEMENT

During a forced hyperflexion injury at the level of the wrist, the second metacarpal is held rigidly in its position because of its constrained osseoligamental environment, preventing dorsal dislocation of the second carpometacarpal joint complex. Subsequent contraction of the extensor carpi radialis longus tendon can only result in an avulsion of the tendon at its insertion, provided that there is sufficient energy during the impact.

In all previously described cases, the cause of the avulsed tendon has been a fall on the flexed hand. This is the first reported case in which the injury is the result of a sports related accident sustained during a rugby head on tackle. During a rugby game, the player in possession 
of the ball often places it close to his body with a flexed elbow and hyperflexed wrist. A forceful impact on the wrist while it is in this position can trigger a contraction of the wrist extensors. Taking into consideration the mechanism involved, we feel that this injury is probably under-reported. In our case, it required a high index of suspicion, as the patient presented in the clinic with minimal symptoms, essentially complaining of a previously unnoted lump at the dorsum of his hand and a relative weakness in dorsiflexion. Furthermore, radiographs did not show the exact anatomy of the fracture, which was revealed only with the assistance of an image intensifier.

Treatment of this type of injury has varied in the literature. Crichlow and Hoskinson ${ }^{3}$ described satisfactory results with conservative management in two of their three cases, whereas Treble and Arif ${ }^{4}$ and De Lee ${ }^{6}$ advocated surgical fixation to maintain the joint congruity and stabilise the fragment. In our experience, conservative management failed to produce an anatomical reduction. The fracture was initially immobilised in its anatomical position, but was noted to have displaced after one week. We believe that this was due to retraction of the muscle while the wrist was immobilised in plaster.

In conclusion, avulsion fracture of the base of the second metacarpal should be suspected in cases of painful restriction of movement of the wrist in a sportsperson involved in a heavy tackle in a contact sport. Radiographic imaging of the avulsed fragment is not always possible, and diagnosis should not depend on it. A high index of clinical suspicion along with a low threshold for operative treatment proved to be the best method of management in our case.

1 Jessa KK, Hodge JC. Avulsion fracture of tendon of extensor carpi radialis longus: unknown mechanism. I Emerg Med 1997;15:201-7.

2 Takami H, Takahashi S, Ando M. Avulsion of the second metacarpal base by the extensor carpi radialis longus. Arch Orthop Trauma Surg 1998;118:109-10.

3 Crichlow TPKR, Hoskinson J. Avulsion fracture of the index metacarpal base: three case reports. F Hand Surg [Br] 1988;13:212-14.

4 Treble N, Arif S. Avulsion fracture of index metacarpal. f Hand Surg [Br] 1987;12:38-9.

5 Sadr B, lalehzarian $M$. Traumatic avulsion of the tendon of extensor carpi radialis longus tendon. $\mathcal{F}$ Hand Surg [Am] 1987;12:1035-7.

6 De Lee JC. Avulsion fracture of base of the second metacarpal by the extensor carpi radialis longus. F Bone foint Surg

7 El-Bacha A. The carpometacarpal joints. In: Tubiana R, ed. The hand. Philadelphia, London and Toronto: WB Saunders Company, 1981:158-68. 